\title{
Categorical Visual Score Traits of a Nellore Beef Cattle Population
}

\author{
Jairo Azevedo Junior ${ }^{1}$, Juliana Petrini², Gerson Barreto Mourão² \& José Bento Sterman Ferraz ${ }^{1}$ \\ ${ }^{1}$ Department of Veterinary Medicine, College of Animal Science and Food Engineering, University of São Paulo, \\ Pirassununga, SP, Brazil \\ ${ }^{2}$ Department of Animal Science, College of Agronomy "Luiz de Queiroz", University of São Paulo, Piracicaba, \\ SP, Brazil \\ Correspondence: Jairo Azevedo Junior, Avenida Pará, 955, Postal Code 77403-010, Gurupi, TO, Brazil. Tel: \\ 55-63-999-336-010. E-mail: jairo@zootecnista.com.br
}

Received: May 3, 2017

Accepted: June 13, 2017 Online Published: July 15, 2017

doi:10.5539/jas.v9n8p63

URL: https://doi.org/10.5539/jas.v9n8p63

\begin{abstract}
Variance components and genetic parameters of economically relevant traits in livestock, whether continuous or categorical, can be estimated by methods computationally available providing support for the selection and mating of animals in breeding programs. The objectives of this paper were to obtain and compare the variance components estimates for visual traits under continuous or categorical distribution in single-trait analysis and their correlations with continuous productive traits in two-trait analysis. Data of conformation (CONF), precocity of fat deposition (PREC) and muscling (MUSC) visual scores evaluated at 18 months of age as well as the weight at 18 months of age (YW) were collected from animals born from 2000 to 2012, in Nellore cattle herds raised in Southeastern and Central Western tropical regions of Brazil. Methods III of Henderson, Restricted Maximum Likelihood (REML), Bayesian Inference and generalized linear mixed model (GLMM) were tested. Variance components obtained from single-trait analysis were similar to those obtained from two-trait analysis. The estimates of heritability $\left(\mathrm{h}^{2}\right)$ for the visual scores ranged from 0.1081 to 0.2190 . Heritability estimates for traits evaluated by visual scores have moderate to high magnitude justifying the inclusion of visual scores as selection criteria in animal breeding and the selection of animals with higher scores for mating. High genetic correlations between yearling weight and morphological traits were verified. For visual scores of conformation, precocity and muscling, the most suitable model based on one-trait or two-trait analyses considered an animal model, a linear distribution of the data and the estimation method of the components of (co)variance based on Bayesian methodology.
\end{abstract}

Keywords: estimation method, genetic analyses, rearing system, variance components, Zebu cattle

\section{Introduction}

Some traits with threshold distribution of data have been more studied and were already included in animal breeding programs for zebu cattle populations, including Nellore cattle. An example is the morphological system based on visual scores such as conformation (CONF), precocity (PREC) and muscling (MUSC) score, which are evaluated at 18 months old. Morphological visual scores have been adopted to estimate the carcass composition and the time when these animals are fit for slaughter (Faria et al., 2010) respecting the fat deposition process and anatomical specifics of Zebu cattle. Through this system a larger number of animals can be evaluated visually without a direct intervention, accelerating the evaluation process and minimizing the stress in the animals. The phenotypes for these morphological traits are grades ranging from one to six or from one to nine depending on the evaluation method adopted.

There are two reasons for the adoption of visual scores as selection criteria: to supply the demand of the consumers by a type of product and to get an indirect response to selection for productivity (Lush, 1964). This indirect response to selection implies in a genetic correlation between morphological visual scores and productive traits. Genetic correlations of high magnitude between these visual scores and weights indicates that selection for conformation, precocity and muscling should also improve animal performance for traits as weaning or yearling weights (Koury Filho et al., 2009, 2010).

Variance components and genetic parameters for categorical traits can be estimated under different approaches, which evolve with the development of new theories and computational techniques. The establishment of an analysis model that correctly describes the data is an important factor in obtaining the genetic parameters (Faria et 
al., 2008). The methodologies vary according to the number of evaluated traits, the nature of the phenotypic data distribution, the genetic structure adopted and the estimation method of the variance components. For example, by the assumption of linear (Gordo et al., 2012; Forutan, Mahyari, \& Sargolzaei, 2015) or threshold distribution of phenotypes (Faria et al., 2009; Boligon et al., 2012; Forutan, Mahyari, \& Sargolzaei, 2015), by using sire or animal models (Forutan, Mahyari, \& Sargolzaei, 2015); and by adopting restricted maximum likelihood (Bouquet et al., 2010; Queiroz et al., 2011), Bayesian (Boligon et al., 2012; Santana Jr. et al., 2013) or generalized linear mixed model approaches (Forutan, Mahyari, \& Sargolzaei, 2015) as estimation methods. These methodologies can be applied in a combined way. However, there is not a consensus of the best approach to perform the genetic analyses of morphological visual scores. Therefore, the aim in this study was to obtain and compare the variance components estimates for visual traits under continuous or categorical distribution in single-trait analysis and their interrelations with continuous productive traits in bi-trait analysis.

\section{Method}

The approval of the Ethics Committee on Animal Use was not necessary in this study because the data were obtained from an existing database.

\subsection{Animals and Data File}

The Nellore cattle data were provided by the Genetic Improvement Program of CFM Ltda. The animals were kept in high quality pastures ( $40 \%$ Brachiaria, Panicum maximum $50 \%$, and $10 \%$ other forage) and received salt and mineral supplement up to 18 months old. The breeding season, 90 days for heifers and 60 days for cows, was concentrate from October to January, depending on the beginning of the rainy season. Calves remained with their dams in high quality pastures until about 7 months old, when they were weaned.

Four traits were analyzed in this study: visual scores of conformation (CONF), precocity (PREC) and muscling (MUSC) and yearling weight (YW, kg). Visual scores traits (CONF, PREC and MUSC) were evaluated at 18 months of age by trained personnel, following the standard procedures established by the Agropecuária CFM, with grades ranging from 1 (unwanted/bad) to 6 (desirable/excellent). The CONF score is a classification of animal's capacity for meat production, considering the combination of quantity of meat in the carcass with the presence of muscle mass. The PREC score is related to the ability of animals to store fat and it is used to identify animals that will deposit covering fat earlier. PREC scores equal 6 indicate animals with more adequate fat reserves. The rating for MUSC considers the muscle mass of the animal, and higher scores are assigned to animals with more muscle mass.

Only data from animals with known parents, with known age of the dam at birth, and animals born between the years 2000 and 2012 were considered. Animals conceived by embryo transfer were excluded. Outliers for age at first calving and YW were detected and eliminated. After discarding records with incomplete and inconsistent data, a description of the traits evaluated in this study is shown in Table 1. Observations recorded between 450 and 650 days of age were considered yearling records.

Contemporary groups (CG) were defined for each trait. For visual scores, two types of CGs were considered: weaning group CG (CG_W), formed by animals reared in the same herd and management group until weaning, and from the same birth year, birth season and sex; and yearling $\mathrm{CG}\left(\mathrm{CG}_{-} \mathrm{Y}\right)$, built with animals reared in the same herd and management group between the weaning and 18 months of age (yearling), from the same birth year, birth season and sex.

Contemporary groups with progenies of only two different bulls and with less than five animals were discarded. It was also discarded CGs with only one phenotypic class. A description of the CGs after their consolidation is listed in Table 1. The distribution of phenotypes for CONF, PREC and MUSC is shown in Figure 1. The database edition was performed by using SAS software (Statistical Analysis System, version 9.3). 
Table 1. Description of data for visuals scores for conformation (CONF), precocity (PREC) and muscling (MUSC), and yearling weight $(\mathrm{YW}, \mathrm{kg}$ ) evaluated in Nellore cattle and number of animals by contemporary group formed for the management group at weaning $\left(\mathrm{CG}_{-} \mathrm{W}\right)$ and at yearling $\left(\mathrm{CG}_{-} \mathrm{Y}\right)$

\begin{tabular}{lllllll}
\hline & CONF & PREC & MUSC & YW & CG_W & CG_Y \\
\hline $\mathrm{N}$ & 53626 & 53626 & 53626 & 53626 & 152 & 286 \\
Mean & 3.40 & 3.47 & 3.30 & 299.94 & 352 & 187 \\
Standard deviation & 1.03 & 1.05 & 1.07 & 46.58 & -- & -- \\
Minimum & 1.00 & 1.00 & 1.00 & 162.00 & 5 & 5 \\
Maximum & 6.00 & 6.00 & 6.00 & 438.00 & 1710 & 1942 \\
\hline
\end{tabular}

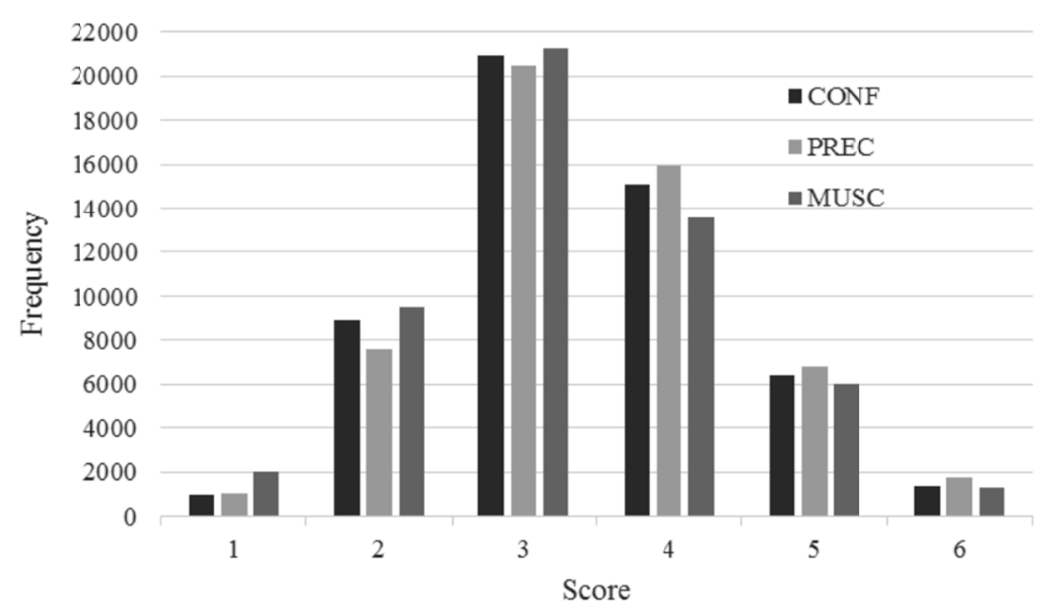

Figure 1. Distribution of records among the scores for conformation (CONF), precocity (PREC) and muscling (MUSC) traits evaluated in Nellore cattle

\subsection{Genetic Analyses}

Estimates of variance components were obtained from different mixed models regarding their genetic structure (sire or animal model), estimation method (Bayesian Inference, Generalized Linear Mixed Models, Restricted Estimated Maximum Likelihood, Method III of Henderson) and the assumed distribution of the data (linear or threshold), as follow:

SHL model: one-trait analysis, (S) sire model, (H) Method III of Henderson, (L) Linear data distribution, performed in SAS;

SRL model: one-trait analysis, (S) sire model, (R) Restricted Estimated Maximum Likelihood, (L) Linear data distribution, performed in ASREML;

ARL model: one-trait analysis, (A) animal model, (R) Restricted Estimated Maximum Likelihood, (L) Linear data distribution, performed in ASREML;

ABL model: one-trait analysis, (A) animal model, (B) Gibbs sampling (Bayesian Inference), (L) Linear data distribution, performed in GIBBS3F90;

ABT model: one-trait analysis, (A) animal model, (B) Gibbs sampling (Bayesian Inference), (T) Threshold data distribution, performed in THRGIBBS3F90;

AGTLo model: one-trait analysis, (A) animal model, (G) Generalized Linear Mixed Models, (TLo) Threshold-Logit data distribution, performed in ASREML;

AGTPr model: one-trait analysis, (A) animal model, (G) Generalized Linear Mixed Models, (TPr) Threshold-Probit data distribution, performed in ASREML;

ARLL model: two-trait analysis, (A) animal model, (R) Restricted Estimated Maximum Likelihood, (LL) Linear-Linear data distribution, performed in ASREML;

ABLL model: two-trait analysis, (A) animal model, (B) Gibbs sampling (Bayesian Inference), (LL) Linear-Linear data distribution, performed in GIBBS3F90; 
ABLT model: two-trait analysis, (A) animal model, (B) Gibbs sampling (Bayesian Inference), (LT) Linear-Threshold data distribution, performed in THRGIBBS3F90.

\subsubsection{Genetic Structure: Sire Model vs. Animal Model}

The animal model considered for the categorical and continuous traits in the genetic analyses under the classic linear model methodology followed the generic mathematical Model (1):

$$
\mathrm{y}=\mathrm{X} \beta+\mathrm{Z}_{1} \mathrm{a}+\mathrm{Z}_{2} \mathrm{c}+\mathrm{e}
$$

Where, $y$ is the vector of response variables; $\beta$ is the vector of fixed effects (AGE and MAP); $a$ is the vector of the random genetic additive direct effects; $\mathrm{c}$ is the vector of random effects of contemporary group at yearling (CG_Y); and e is the vector of random residual effects inherent to each observation; $\mathrm{X}, \mathrm{Z}_{1}$ and $\mathrm{Z}_{2}$ represent the incidence matrices for fixed and random effects, associating respectively the elements of $\beta$, a and $c$ to the response variable.

The assumptions for the model were: $\mathrm{E}[\mathrm{y}]=\mathrm{X} \beta ; \mathrm{E}[\mathrm{a}]=0 ; \mathrm{E}[\mathrm{c}]=0 ; \mathrm{E}[\mathrm{e}]=0$; and

$$
\mathrm{V}\left[\begin{array}{l}
\mathrm{a} \\
\mathrm{c} \\
\mathrm{e}
\end{array}\right]=\left[\begin{array}{ccc}
\mathrm{A} \sigma_{\mathrm{a}}^{2} & 0 & 0 \\
0 & \mathrm{I} \sigma_{\mathrm{c}}^{2} & 0 \\
0 & 0 & \mathrm{I} \sigma_{\mathrm{e}}^{2}
\end{array}\right]
$$

Where, A is the matrix of genetic covariances among animals; I is the identity matrix; $\sigma_{a}^{2}$ is the genetic additive direct variance; $\sigma_{c}^{2}$ is the variance due to the effects of contemporary group at yearling; and $\sigma_{e}^{2}$ is the residual variance. The covariances between all the effects were assumed to be zero. In the sire model the animal direct effect was replaced by the direct effect of the sire.

\subsection{Estimation Methods}

Estimation methods evolve with the development of new theories and computational techniques. It was analyzed four different estimation methods in this study: Method III of Henderson, Method of Restricted Estimated Maximum Likelihood (REML), Method of Generalized Linear Mixed Models (GLMM) and Gibbs sampling (Bayesian inference).

Method III of Henderson (Henderson, 1953) is the simplest and oldest of them. This method was widely used until the 80's and does not use the relationship matrix to solve the mixed effects. Also called method of fitting constants, it uses the reductions in the sums of squares of the complete model and of the sub-models to find the estimators for the variance components, setting up a system of equations from the differences between the reductions in the full model and sub-models, equating them to their respective expectation. According to Henderson (1953) method III can be used for any mixed model and produces unbiased estimates of the variance components. In this study, Method III of Henderson was performed by using SAS software (Statistical Analysis System, version 9.3).

Method of Restricted Estimated Maximum Likelihood (REML) was proposed by Patterson and Thompson (1971) based on previous studies of Fischer (1925), and Hartley and Rao (1967). In REML, each observation is divided in two independent parts, the fixed and random effects, and the probability density function of the observations is given by the sum of the probability density functions of each part. The maximization of the probability density function portion related to random effects in relation to the variance components eliminates the bias resulting of the loss of degrees of freedom in the estimation of fixed effects. When REML equations include balanced data this method is identical to ANOVA estimators being non-biased and with minimum variance. However, when the REML estimator takes into account the degrees of freedom involved in the estimation of fixed effects, and when REML is applied to unbalanced data, REML estimators can be biased (Searle, 1987). The REML estimators for each variance component are a function of estimates of the other components, and can only be found by iterative numerical methods. In this study, variance components obtained by REML methodology were obtained from analyses based on sire model and animal model by using AIREMLF90 (Misztal et al., 2015) and ASREML v4.1 (Gilmour et al., 2015) software.

Method of Generalized Linear Mixed Models (GLMM, Nelder \& Wedderburn, 1972) is an extension of linear models and constitute a number of techniques commonly studied separately. The models involve three components: a variable response, explanatory variables and a random sample of $n$ observations. Systematic effects (fixed and random effects) are linearized by a function of the expected values (for example, Log, Probit and Logit functions), allowing fitted values varying in the response amplitude. In this study the variance components were obtained assuming a threshold distribution for the data and using ASREML program (Gilmour 
et al., 2015) with the link functions Probit and Logit. The residual variance for the Logit function was set at 3.289; and the residual variance in the Probit function was set at 1 .

One of the newest approach for estimation methods is the Gibbs sampling (Gianola \& Foulley, 1983) based on the Bayesian inference. Some difficulties can be verified in obtaining Gibbs chain convergence in animal models because the algorithm used is characterized as an iterative process (Faria et al., 2008). Nevertheless, Bayesian inference is recommended to obtain genetic correlations between categorical (survival and visual scores) and continuous traits (weights) through multi-trait analysis (Everling et al., 2014). In this study, the Bayesian approach was performed using the GIBBS3F90 (Misztal et al., 2015) program (assuming linear distribution of data) and THRIGIBBS3F90 (Misztal et al., 2015) program (assuming threshold distribution of data). A total of 1 000000 iterations were performed, with sampling interval every 10 interactions and burn in of 10000 iterations. Analyses of subsequent estimates were performed using the POSTGIBBS3F90 (Misztal et al., 2015) program.

The analysis models and their effects (fixed and random) proposed for each trait are described in Table 2.

Table 2. Summary of the models adjusted for the estimation of (co)variance components for the conformation (CONF), precocity (PREC) and muscling (MUSC) scores and body weight (YW) measured in Nellore cattle at 18 months of age

\begin{tabular}{llllll}
\hline Trait & ANI & CG_W & CG_Y & AGE & MAP \\
\hline CONF & R & R & F & F & F \\
PREC & R & R & F & F & F \\
MUSC & R & R & F & F & F \\
YW & R & R & F & F & F \\
\hline
\end{tabular}

Note. ANI $=$ genetic additive direct effect; $\mathrm{CG}_{-} \mathrm{W}=$ management group at weaning; CG_Y = contemporary group at yearling; $\mathrm{AGE}=$ real age of the animal at yearling (linear and quadratic effects covariates); MAP = mother's age at calving (linear and quadratic effects covariates). $\mathrm{R}=$ random effect; $\mathrm{F}=$ fixed effect.

\subsection{Heritability Coefficient Estimates}

The heritability estimates for CONF, PREC, MUSC and YW traits were obtained from the Equation (3):

$$
\mathrm{h}_{\mathrm{a}}^{2}=\left(\sigma_{\mathrm{a}}^{2}\right) /\left(\sigma_{\mathrm{a}}^{2}+\sigma_{\mathrm{CG}_{-} \mathrm{Y}}^{2}+\sigma_{\mathrm{e}}^{2}\right)
$$

Where, $h_{\mathrm{a}}{ }^{2}$ is the narrow sense heritability, $\sigma_{\mathrm{a}}^{2}$ is the additive variance, $\sigma_{\mathrm{CG}_{-} \mathrm{Y}}^{2}$ is the variance due to the effects of contemporary group at yearling, and $\sigma_{\mathrm{e}}^{2}$ is the residual variance.

In the sire model analyses, the heritabilities for CONF, PREC, MUSC and YW traits from the Equation (4):

$$
\mathrm{h}_{\mathrm{a}}^{2}=\left(4 \sigma_{\mathrm{s}}^{2}\right) /\left(\sigma_{\mathrm{s}}^{2}+\sigma_{\mathrm{CG} \_\mathrm{Y}}^{2}+\sigma_{\mathrm{e}}^{2}\right)
$$

Where, $h_{a}{ }^{2}$ is the narrow sense heritability, $\sigma_{\mathrm{s}}^{2}$ is the sire variance, $\sigma_{\mathrm{CG}_{-} \mathrm{Y}}^{2}$ is the variance due to contemporary group at yearling effects, and $\sigma_{\mathrm{e}}^{2}$ is the residual variance.

\subsection{Genetic Correlation Estimates}

Genetic correlations between visual scores (CONF, PREC or MUSC ) and YW traits estimated in animal model and two-trait analyses were performed for ARLL, ABLL and ABLT models to compare the Bayesian inference and REML estimation methods and the linear and threshold data distribution. Estimates of additive genetic correlations $\left(\mathrm{r}_{\mathrm{a}}\right)$ were obtained from the Equation (5):

$$
r_{a}=\sigma_{\mathrm{a}_{(\mathrm{VISUAL}, \mathrm{YW})}} /\left(\sqrt{\sigma_{\mathrm{a}_{(\mathrm{VISUAL})}^{2}}^{2}} \times \sqrt{\sigma_{\mathrm{a}_{(\mathrm{YW})}^{2}}^{2}}\right)
$$

Where, $\sigma_{\mathrm{a}(\mathrm{VISUAL}, \mathrm{YW})}$ is the covariance between additive direct effects of visual scores and $\mathrm{YW}$ traits, $\sigma_{\mathrm{a}(\mathrm{VISUAL})}^{2}$ is the visual score additive variance and $\sigma_{\mathrm{a}(\mathrm{YW})}^{2}$ is the $\mathrm{YW}$ additive variance.

\section{Results and Discussion}

The observed means verified in this study for CONF, PREC and MUSC (Table 1) were 3.40, 3.47 and 3.30, respectively - similar to those reported in previous studies. Considering Nellore cattle at yearling age, Lima et al. (2013), Santana, Jr. et al. (2013), Faria et al. (2010) and Koury Filho et al. (2009) found mean scores for PREC of $3.87,3.66,3.86$ and 3.85, respectively. For MUSC, the authors cited above found mean scores of 3.77, 3.57, 3.62 and 3.80 , respectively. 
Variance components for CONF, MUSC, and PREC obtained by the adjustment of different models are shown in Table 3. There was a convergence of the models, including those adjusted with Bayesian Inference. The posterior distributions of the heritability estimated by using Bayesian Inference are presented in Figures 2, 3 and 4 for CONF, PREC and MUSC traits, respectively. In these figures it is possible to verify the convergence of the Gibbs chain with symmetric Bayesian posterior distributions, similar to a normal distribution according to Van Tassell, Van Vleck, and Gregory (1998). The good definition of contemporary groups and the number of observations available in each class of contemporary groups contribute to explain the good convergence of the Gibbs chain.

The inclusion of the additive relationship matrix between animals in the SRL model did not reduced the variance associated to the sire effect and the heritabilities for PREC, CONF and MUSC. However, the inclusion of the additive relationship matrix can increase the reliability associated to these estimates. The heritability estimated by using SHL and SRL models was lower than the estimates obtained through the animal models. The structure of genetic variances and covariance established through the relationship matrix allows a better isolation of the genetic effect. Accordingly, the additive variance and consequently the heritability are greater, indicating that the selection based on own individual records can contribute in a better response to selection.

Table 3. Variance component and heritability estimates for conformation score (CONF), precocity score (PREC) and muscling score (MUSC) evaluated at 18 months of age in a Nellore cattle population, estimated by different methodologies

\begin{tabular}{llllll}
\hline Model $^{1}$ & \multicolumn{1}{c}{$\sigma_{\mathrm{s}}^{2}$} & \multicolumn{1}{c}{$\sigma_{\mathrm{a}}^{2}$} & $\sigma_{\mathrm{CG} \_\mathrm{Y}}^{2}$ & \multicolumn{1}{c}{$\sigma_{\mathrm{e}}^{2}$} & $\mathrm{~h}_{\mathrm{a}}{ }^{2}$ \\
\hline CONF & & & & & \\
SHL & 0.0229 & - & 0.0039 & 0.7325 & 0.1206 \\
SRL & 0.0206 & - & 0.0047 & 0.7377 & $0.1079(0.0107)$ \\
ARL & - & 0.1321 & 0.0047 & 0.6388 & $0.1703(0.0097)$ \\
ABL & - & 0.1324 & 0.0050 & 0.6381 & $0.1708(0.0104)$ \\
ABT & - & 0.0405 & 0.0027 & 0.3700 & $0.1081(0.0340)$ \\
AGTLo & - & 0.7356 & 0.0271 & 3.289 & $0.1816(0.0058)$ \\
AGTPr & - & 0.1934 & 0.0082 & 1.00 & $0.1609(0.0060)$ \\
\hdashline$P R E C$ & & & & & \\
SHL & 0.0346 & - & 0.0037 & 0.8037 & 0.1644 \\
SRL & 0.0321 & - & 0.0047 & 0.8079 & $0.1520(0.0126)$ \\
ARL & - & 0.1672 & 0.0045 & 0.6827 & $0.1957(0.0099)$ \\
ABL & - & 0.1674 & 0.0048 & 0.6820 & $0.1960(0.0104)$ \\
ABT & - & 0.0531 & 0.0027 & 0.3714 & $0.1363(0.0407)$ \\
AGTLo & - & 0.8499 & 0.0241 & 3.289 & $0.2042(0.0057)$ \\
AGTPr & - & 0.2281 & 0.0077 & 1.00 & $0.1846(0.0059)$ \\
\hdashline$M U S C$ & & & & & \\
SHL & 0.0375 & - & 0.0042 & 0.8217 & 0.1737 \\
SRL & 0.0366 & - & 0.0051 & 0.8266 & $0.1687(0.0133)$ \\
ARL & - & 0.1724 & 0.0049 & 0.6987 & $0.1967(0.0099)$ \\
ABL & - & 0.1727 & 0.0057 & 0.6980 & $0.1971(0.0103)$ \\
ABT & - & 0.0661 & 0.0036 & 0.4105 & $0.1484(0.0396)$ \\
AGTLo & - & 0.9296 & 0.0261 & 3.289 & $0.2190(0.0057)$ \\
AGTPr & - & 0.2329 & 0.0082 & 1.00 & $0.1876(0.0059)$ \\
\hline
\end{tabular}

Note. $\sigma_{\mathrm{s}}^{2}$ sire variance; $\sigma_{\mathrm{a}}^{2}$ animal variance; $\sigma_{\mathrm{CG}}^{2} \mathrm{Y}$ variance due to contemporary group at yearling effects; $\sigma_{\mathrm{e}}^{2}$ is the residual variance; $\mathrm{h}_{\mathrm{a}}{ }^{2}$ : narrow sense heritability. ${ }^{1}$ Models composition: (S) sire model, (A) animal model, (H) Method III of Henderson, (R) Restricted Estimated Maximum Likelihood, (B) Gibbs sampling (Bayesian Inference), (G) Generalized Linear Mixed Models, (L) Linear data distribution, (T) Threshold data distribution, (TLo) Threshold-Logit data distribution, (TPr) Threshold-Probit data distribution.

In animal models, the lowest (co) variance components and genetic parameters for CONF, PREC and MUSC (Table 3) were obtained by adjusting the ABT model, and the highest estimates were obtained with the adjustment of the AGTLo model. Therefore, it is observed that the ABT and AGTLo models appear to be more influenced by 
the number of levels/phenotypic classes of categorical traits. For ABT the lowest heritability estimate results in an increase of standard error, indicating a possible bias in the iterative process under an animal model and an underestimation of (co)variance components. Hoeschele et al. (1995) also reports difficulties to adjustment of Bayesian inference in this case where the Gibbs sampler may not converge under animal model. In a parallel investigation (data not showed) a process with 1500000 iterations was performed and the (co)variance components did not changed. Contrasting to the others models the AGTLo results in highest heritabilities estimates associated to the lowest adjusted standard error.

(a)

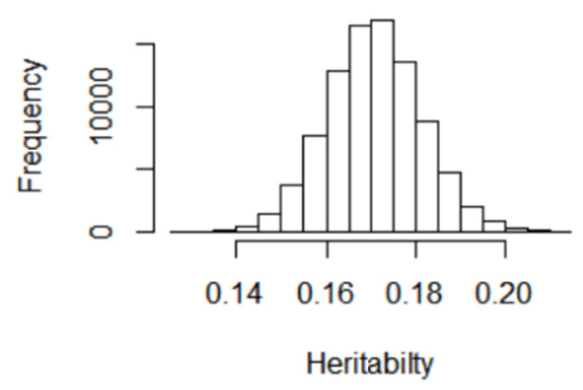

(b)

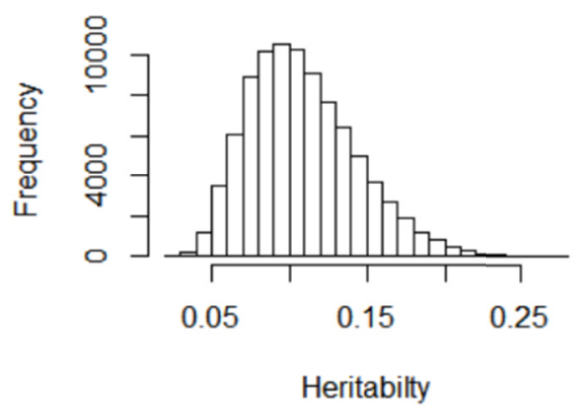

Figure 2. Distribution of the posterior estimates of direct heritability for conformation score (CONF) measured in Nellore cattle at 18 months of age assessed under ABL (a) and ABT (b) models

(a)

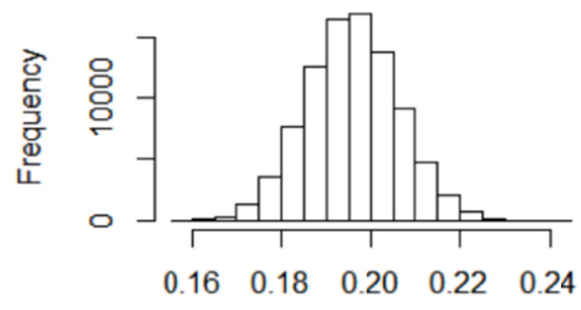

Heritability (b)

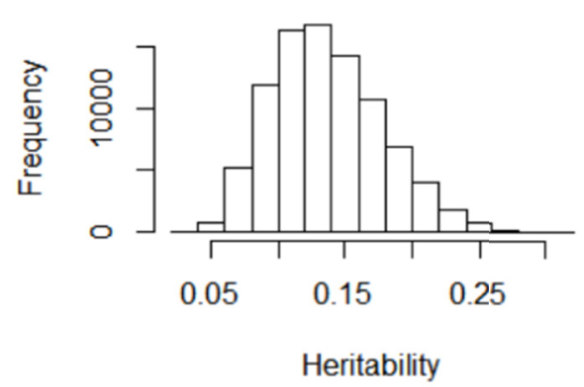

Figure 3. Distribution of the posterior estimates of direct heritability for precocity score (PREC) measured in Nellore cattle at 18 months of age assessed under the ABL (a) and ABT (b) models

(a)

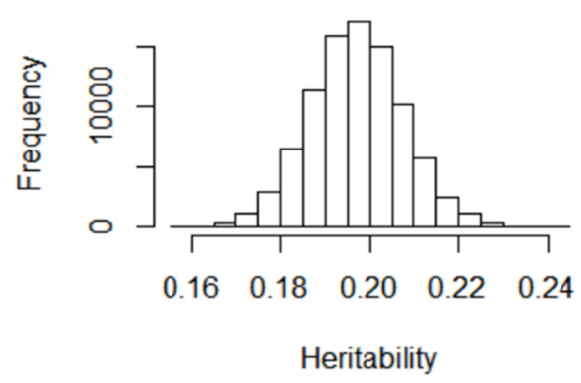

(b)

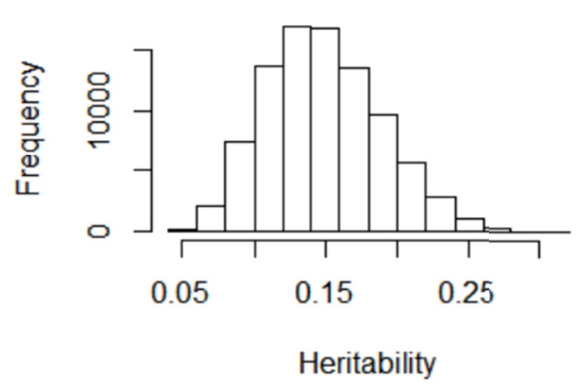

Figure 4. Distribution of the posterior estimates of direct heritability for muscling score (MUSC) measured in Nellore cattle at 18 months of age assessed under the ABL (a) and ABT (b) models 
The heritability estimated for CONF (Table 3 ) ranged from 0.1079 to 0.1816 , for PREC between 0.1363 and 0.2042 , and for MUSC from 0.1484 to 0.2190 . Thus all the estimates were of moderate magnitude they were similar to those reported in previous studies. Koury Filho et al. (2010) reported heritability estimates for CONF, PREC and MUSC evaluated in yearling beef cattle of $0.24,0.32$ and 0.27 , respectively. Estimates of heritability of 0.26 for PREC and MUSC traits analyzed under a multi-trait linear threshold Bayesian approach were reported by Santana Jr et al. (2013). Pedrosa et al. (2010) and Araújo et al. (2010) estimated heritabilities for CONF of 0.23 and 0.16 , respectively, 0.19 and 0.17 to PREC, and 0.22 and 0.16 for MUSC. Differences in magnitude of the heritability coefficients of visual scores may be associated with differences in the measurement systems and models used in the analyses of these traits (Cardoso, Cardellino, \& Campos, 2004).

Evaluating conformation and muscularity in Nellore beef cattle, Farias et al. (2008) found that there is no difference between the linear model and the threshold model in the estimation of heritability and genetic correlations for multinomial categorical morphological traits. In this study, there were differences between the above models for CONF, PREC and MUSC, with higher heritability estimates obtained through linear models in comparison with the threshold models (Table 3, models ABL vs ABT). Ducrocq et al. (1988) stated that the threshold methods may have greater ability to detect the genetic variability than the linear methods. In this study, the highest variance components and heritability estimates were obtained with the linear model, but associated with a higher residual error. A possible explanation for higher estimates in linear model is the number of levels considered for the morphological visual scores, varying from 1 to 6 and, therefore, tending to a normal distribution. However, independently of the model and methodology used, the estimates of heritability obtained are indicative of the possibility to achieve positive genetic changes in carcass and meat quality in this population by adopting visual scores as selection criteria.

Similar estimates of heritability were obtained for CONF, PREC and MUSC by using REML and Bayesian estimation methods under animal model and linear distribution of data were (Table 3, models ARL vs. ABL). Previous studies that used animal models for analyses with categorical data (Luo, Boettcher, \& Schaeffer, 2001; Phocas \& Laloe, 2003) reported difficulty to achieve Gibbs chain convergence chain, which was not observable in this study as verified in Figures 2, 3 and 4.

As cited above, higher additive variance and heritability estimates were verified for CONF, PREC and MUSC (Table 3) in the AGTLo model and similar estimates was verified for the AGTPr model. The higher additive variances can be related to the fixed residual variance in GLMM procedure.

The average weight of calves at yearling found in this study (Table 1) was lower than that reported by Koury Filho et al. (2009), Yokoo et al. (2010), Lima et al. (2013) and Araújo et al. (2014), which reported average weights adjusted for 550 days of $330.91,347.14,354.71$ and $322.12 \mathrm{~kg}$, respectively.

The variance components estimates obtained in the two-trait analyses for CONF, PREC and MUSC (Table 4) were higher than those obtained in the one-trait analyses. The heritability estimates for yearling weight were similar for all models evaluated, ranging from 0.3459 to 0.3497 , indicating similarity among the different estimation methods for this trait and allowing comparisons among the methods for each of the visual scores traits. According to Meyer (1991) this increase in the estimates of heritability, especially in the additive genetic variance component, may reflect the reduction of the existing bias in analyses that consider only the performance of a trait individually.

Garnero et al. (2010) estimated heritability for yearling weight of 0.26 and Araújo et al. (2014) of 0.38 based on the REML methodology. In contrast, Santos et al. (2012) and Oliveira Jr et al. (2014) found heritability estimates of 0.41 and 0.40 for yearling weight, respectively, which are values higher than those observed in this study.

Genetic correlations between YW and CONF, PREC and MUSC (Table 4) were positive and of high magnitude, indicating that sire selection for visual scores will also result in genetic gain for YW. The highest genetic correlations were, in descending order, observed between YW and CONF, YW and PREC and YW and MUSC. Koury Filho et al. (2010) reported correlations between YW and CONF, PREC and MUSC ranging from 0.58 to 0.83. Lima et al. (2013) reported correlations between YW and PREC and between YW and MUSC of 0.83 and 0.91 , respectively, confirming the estimates of genetic correlations observed in this study. Biologically, high genetic correlations suggest a gene with pleiotropic effect controlling the traits expression. Pereira et al. (2016) verified a pleiotropic effect of the pleomorphic adenoma gene 1 (PLAG1), in a network involved in the modulation of the function and expression of IGF1 (insulin like growth factor 1), IGF2 (insulin like growth factor 2), GH1 (growth hormone 1), IGF1R (insulin like growth factor 1 receptor) and GHR (growth hormone receptor), which are well known major actors of the growth pathway. 
Table 4. Variance components and heritability estimates for conformation (CONF), precocity (PREC) and muscling (MUSC) score and yearling weight (YW) evaluated at 18 months in a Nellore cattle population, estimated by using different methodologies in two-trait analyses

\begin{tabular}{llrlrlll}
\hline Model $^{1}$ & Trait & $\sigma_{a}^{2}$ & $\sigma_{C G_{Y} Y}^{2}$ & $\sigma_{e}^{2}$ & $\mathrm{~h}_{\mathrm{a}}{ }^{2}$ & \multicolumn{1}{l}{$\operatorname{LogL}$} & $\mathrm{r}_{\mathrm{a}}$ \\
\hline ARLL & CONF & 0.1503 & 0.0058 & 0.6254 & $0.1923(0.0092)$ & -251892 & $0.9362(0.0087)$ \\
& YW & 226.94 & 18.815 & 403.49 & $0.3495(0.0115)$ & & \\
ABLL & CONF & 0.1510 & 0.0063 & 0.6250 & $0.1930(0.0101)$ & -243799 & $0.9357(0.0090)$ \\
& YW & 227.20 & 20.597 & 403.40 & $0.3489(0.0121)$ & & \\
ABLT & CONF & 0.0059 & 0.0002 & 0.0281 & $0.1750(0.0265)$ & -189279 & $0.8801(0.0167)$ \\
& YW & 227.60 & 18.994 & 404.10 & $0.3497(0.0126)$ & & \\
ARLL & PREC & 0.1740 & 0.0059 & 0.6775 & $0.2029(0.0097)$ & -255915 & $0.7484(0.0164)$ \\
& YW & 224.57 & 18.425 & 404.95 & $0.3466(0.0114)$ & & \\
ABLL & PREC & 0.1745 & 0.0064 & 0.6772 & $0.2033(0.0103)$ & -245768 & $0.7479(0.0172)$ \\
& YW & 224.80 & 20.190 & 404.90 & $0.3459(0.0120)$ & & \\
ABLT & PREC & 0.0073 & 0.0005 & 0.0316 & $0.1919(0.0317)$ & -199027 & $0.7017(0.0233)$ \\
& YW & 226.00 & 19.613 & 405.20 & $0.3473(0.0125)$ & & \\
ARLL & MUSC & 0.1784 & 0.0065 & 0.6940 & $0.2030(0.0096)$ & -256983 & $0.7166(0.0175)$ \\
& YW & 224.68 & 18.306 & 404.85 & $0.3468(0.0114)$ & & \\
ABLL & MUSC & 0.1789 & 0.0071 & 0.6937 & $0.2033(0.0102)$ & -246424 & $0.7163(0.0182)$ \\
& YW & 224.90 & 20.046 & 404.80 & $0.3461(0.0120)$ & & \\
ABLT & MUSC & 0.0165 & 0.0012 & 0.0501 & $0.2476(0.0301)$ & -202588 & $0.6272(0.0232)$ \\
& YW & 225.80 & 19.740 & 404.40 & $0.3473(0.0124)$ & & \\
\hline
\end{tabular}

Note. $\sigma_{\mathrm{s}}^{2}$ : sire variance; $\sigma_{\mathrm{a}}^{2}$ : animal variance; $\sigma_{\mathrm{CG}}^{2}$ : variance due to contemporary group at yearling effects; $\sigma_{\mathrm{e}}^{2}$ : is the residual variance; $h_{\mathrm{a}}{ }^{2}$ : narrow sense heritability; LogL: Likelihood function logaritm; $r_{\mathrm{a}}$ : genetic correlation.

${ }^{1}$ Models composition: (A) animal model, (R) Restricted Estimated Maximum Likelihood, (B) Gibbs sampling (Bayesian Inference), (LL) Linear-Linear data distribution, (LT) Linear-Threshold data distribution.

Considering the likelihood function logarithm in two-trait analyses (Table 4) the lowest values were verified for the ABLT model suggesting better adjustment according Bayesian inference and visual scores considered as categorical data distribution. However, the lowest LogL for ABLT is associated to lowest heritabilities and genetic correlation estimates in this model. Weighting between higher heritabilities and genetic correlation and lower likelihood function logarithm the ABLL can be consider a reasonable approach to modeling the genetic analyses for these morphological traits.

\section{Conclusion}

Heritability estimates for traits evaluated by visual scores have medium to high magnitude justifying the selection of animals with higher scores for mating. High genetic correlations between yearling weight and morphological traits were verified. For visual scores of conformation, precocity and muscling, the most suitable model based on one-trait or two-trait analyses considered an animal model, a linear distribution of the data and the estimation method of the components of (co)variance based on Bayesian methodology.

\section{Acknowledgements}

To the Agropecuaria CFM Ltda for providing the data and information used in this study, the University of São Paulo by the structure necessary for the development of this project. To the Coordenação de Aperfeiçoamento de Pessoal de Nível Superior-CAPES (process 3048/2010), Fundação de Amparo à Pesquisa do Estado de São Paulo-FAPESP (process 2012/50533-2 and 2014/07566-2) and Conselho Nacional de Desenvolvimento Científico e Tecnológico-CNPq for financial support.

\section{References}

Araújo, C. V. D., Lôbo, R. B., Figueiredo, L. G. G., Mousquer, C. J., Laureano, M. M. M., Bittencourt, T. C. B. D. S., \& Araújo, S. I. (2014). Estimates of genetic parameters of growth traits of Nellore cattle in the Midwest region of Brazil. Revista Brasileira de Saúde e Produção Animal, 15(4), 846-853. https://doi.org/10.1590/ S1519-99402014000400006 
Boligon, A. A., Ayres, D. R., Pereira, R. J., Morotti, N. P., \& Albuquerque, L. G. (2012). Genetic associations of visual scores with subsequent rebreeding and days to first calving in Nellore cattle. Journal of Animal Breeding and Genetics, 129(6), 448-456. https://doi.org/10.1111/j.1439-0388.2012.00998.x

Bouquet, A., Fouilloux, M. N., Renand, G., \& Phocas, F. (2010). Genetic parameters for growth, muscularity, feed efficiency and carcass traits of young beef bulls. Livestock Science, 129(1-3), 38-48. https://doi.org/10.1016/ j.livsci.2009.12.010

Cardoso, F. F., Cardellino, R. A., \& Campos, L. T. (2004). (Co)variance components and genetic parameters of post-weaning traits in Angus cattle. Brazilian Journal of Animal Science, 33(2), 313-319. http://dx.doi.org/ $10.1590 /$ S1516-35982004000200006

Ducrocq, V., Quaas, R. L., Pollak, E. J., \& Casella, G. (1988). Length of productive life of dairy cows. 2. Variance component estimation and sire evaluation. Journal of Dairy Science, 71(11), 3071-3079. https://doi.org/10.3168/jds.S0022-0302(88)79907-5

Everling, D. M., Bresolin, T., Rorato, P. R., Araujo, R. O., Boligon, A. A., Weber, T., ... Campos, L. T. (2014). Finishing precocity visual score and genetic associations with growth traits in Angus beef cattle. Genetics and Molecular Research, 13(3), 7757-776. https://doi.org/10.4238/2014.September.26.13

Faria, C. U., Iwres, B. C., Vozzi, A. P., Magnabosco, C. U., Koury Filho, W., Viu, M. A., ... Lôbo, R. B. (2010). Genetic correlations between categorical morphological traits in Nellore cattle by applying Bayesian analysis under a threshold animal model. Journal of Animal Breeding and Genetics, 127(5), 377-384. https://doi.org/10.1111/j.1439-0388.2010.00860.x

Faria, C. U., Magnabosco, C. U., Albuquerque, L. G., Bezerra, L. A. F., \& Lôbo, R. B. (2009). Genetic correlation estimates between visual scores and carcass traits measured by ultrasound in Nellore cattle using linear threshold Bayesian models. Brazilian Journal of Animal Science, 38(11), 2144-2151. https://doi.org/10.1590/ S1516-35982009001100011

Faria, C. U., Magnabosco, C. U., Albuquerque, L. G., Los Reyes, A., Bezerra, L. A. F., \& Lôbo, R. B. (2008). Genetic analysis for visual scores of bovines with the linear and threshold Bayesian models. Pesquisa Agropecuária Brasileira, 43(7), 835-841. https://doi.org/10.1590/S0100-204X2008000700007

Forutan, M., Mahyari, S. A., \& Sargolzaei, M. (2015). Genetic evaluation of calf and heifer survival in Iranian Holstein cattle using linear and threshold models. Journal of Animal Breeding and Genetics, 132(1), 51-58. https://doi.org/10.1111/jbg.12115

Garnero, A. V., Lôbo, R. B., Bezerra, L. A. F., \& Oliveira, H. N. (2010). Comparison between some selection criteria for growth traits correlated to scrotal circumference. Brazilian Journal of Animal Science, 30(3), 714-718. https://doi.org/10.1590/S1516-35982001000300016

Gianola, D., \& Foulley, J. L. (1983). Sire evaluation for ordered categorical data with a threshold model. Genetics Selection Evolution, 15(2), 201-224. https://doi.org/10.1186/1297-9686-15-2-201

Gilmour, A. R., Gogel, B. J., Cullis, B. R., Welham, S. J., \& Thompson, R. (2015). ASReml User Guide Release 4.1 Functional Specification. VSN International Ltd., Hempstead, UK.

Gordo, D. G. M., Baldi, F., Lôbo, R. B., Koury Filho, W., Sainz, R. D., \& Albuquerque, L. G. (2012). Genetic association between body composition measured by ultrasound and visual scores in Brazilian Nellore cattle. Journal of Animal Science, 90(12), 4223-4229. https://doi.org/10.2527/jas.2011-3935

Hartley, H. O., \& Rao, J. N. K. (1967). Maximum likelihood estimation for the mixed analysis of variance model. Biometrika, 54(1-2), 93-108. https://doi.org/10.1093/biomet/54.1-2.93

Henderson, C. R. (1953). Estimation of variance and covariance components. Biometrics, 9(2), 226-252. https://doi.org/10.2307/3001853

Koury Filho, W., Albuquerque, L. G., Alencar, M. M., Forni, S., Silva, J. A. V., \& Lôbo, R. B. (2009). Estimates of heritabilities and correlations for visual scores, weight and height at 550 days of age in Nellore cattle herds. Brazilian Journal of Animal Science, 38(12), 2362-2367. https://doi.org/10.1590/S1516-35982009001 200010

Koury Filho, W., Albuquerque, L. G., Forni, S., Silva, J. A. V., Yokoo, M. J., \& Alencar, M. M. (2010). Genetic parameters estimates for visual scores and their association with body weight in beef cattle. Brazilian Journal of Animal Science, 39(5), 1015-1022. https://doi.org/10.1590/S1516-35982010000500011 
Lima, P. R., Paiva, S. R., Cobuci, J. A., Braccini Neto, J., Machado, C. H., \& McManus, C. (2013). Genetic parameters for type classification of Nellore cattle on central performance tests at pasture in Brazil. Tropical Animal Health and Production, 45(7), 1627-34. https://doi.org/10.1007/s11250-013-0408-1

Luo, M. F., Boettcher, P. J., \& Schaeffer, L. R. (2001). Bayesian inference for categorical traits with an application to variance component estimation. Journal of Dairy Science, 84(3), 694-704. https://doi.org/10.3168/ jds.S0022-0302(01)74524-9

Meyer, K. (1991). Estimating variances and covariances for multivariate animal models by restricted maximum likelihood. Genetics Selection Evolution, 23, 67-83. https://doi.org/10.1186/1297-9686-23-1-67

Misztal, I., Tsuruta, S., Lourenço, D., Aguilar, I., Legarra, A., \& Vitezica, Z. (2015). Manual for BLUPF90 family of programs. University of Georgia, Athens, GA, USA.

Nelder, J. A., \& Wedderburn, R. W. M. (1972). Generalized Linear Models. Journal of the Royal Statistical Society: Series A (Statistics in Society), 135(3), 370-384. https://doi.org/10.2307/2344614

Oliveira Júnior, G. A., Eler, J. P., Ramírez-Díaz, J., Ferraz, J. B. S., \& Santana Jr, M. L. (2014). Inclusion of weaning management group as a random effect in the genetic evaluation of postweaning traits in Nellore cattle. Tropical Animal Health and Production, 46(6), 1031-1036. https://doi.org/10.1007/s11250-0140607-4

Patterson, H. D., \& Thompson, R. (1971). Recovery of inter-block information when block sizes are unequal. Biometrika, 58(3), 545-554. https://doi.org/10.1093/biomet/58.3.545

Pedrosa, V. B., Eler, J. P., Ferraz, J. B. S., \& Pinto, L. F. B. (2014). Utilization of single-trait and multi-trait models applied to genetic parameter estimation in Nellore Cattle. Arquivo Brasileiro de Medicina Veterinária e Zootecnia, 66(6), 1802-1812. https://doi.org/10.1590/1678-6170

Pereira, A. G. T., Utsunomiya, Y. T., Milanesi, M., Torrecilha, R. B. P., Carmo, A. S., Neves, H. H. R., ... Garcia, J. F. (2016). Pleiotropic Genes Affecting Carcass Traits in Bos indicus (Nellore) Cattle Are Modulators of Growth. PLoS ONE, 11(7), e0158165. https://doi.org/10.1371/journal.pone.0158165

Phocas, F., \& Laloe, D. (2003). Evaluation models and genetic parameters for calving difficulty in beef cattle. Journal of Animal Science, 81(4), 933-938. https://doi.org/10.2527/2003.814933x

Queiroz, S. A., Oliveira, J. A., Costa, G. Z., \& Fries, L. A. (2011). Estimates of genetic parameters for visual scores and daily weight gain in Brangus animals. Animal, 5(6), 838-843. https://doi.org/10.1017/S175173 1110002442

Santana Jr, M. L., Eler, J. P., Cucco, D. C., Bignardi, A. B., \& Ferraz, J. B. S. (2013). Genetic associations between hip height, body conformation scores, and pregnancy probability at 14 months in Nellore cattle. Livestock Science, 154(1-3), 13-18. https://doi.org/10.1016/j.livsci.2013.02.018

Santos, G. C. J., Lopes, F. B., Marques, E. G., Silva, M. C., Cavalcante, T. V., \& Ferreira, J. L. (2012). Genetic trend for adjusted weight at 205, 365 and 550 days of age of Nellore cattle from Northern Brazil. Acta Scientiarum. Animal Sciences, 34(1), 97-101. https://doi.org/10.4025/actascianimsci.v34i1.12172

Searle, S. R. (1987). Linear Models for Unbalanced data. John Wiley, New York, NY, USA.

Van Tassell, C. P., Van Vleck, L. D., \& Gregory, K. E. (1998). Bayesian analysis of twinning and ovulation rates using a multiple: Trait threshold model and Gibbs sampling. Journal of Animal Science, 76, 2048-2061. https://doi.org/10.2527/1998.7682048x

Yokoo, M. J., Lobo, R. B., Araujo, F. R. C., Bezerra, L. A. F., Sainz, R. D., \& Albuquerque, L. G. (2010). Genetic associations between carcass traits measured by real-time ultrasound and scrotal circumference and growth traits in Nellore cattle. Journal of Animal Science, 88(1), 52-58. https://doi.org/10.2527/jas.2008-1028

\section{Copyrights}

Copyright for this article is retained by the author(s), with first publication rights granted to the journal.

This is an open-access article distributed under the terms and conditions of the Creative Commons Attribution license (http://creativecommons.org/licenses/by/4.0/). 